\title{
Asociación del polimorfismo FecB con la prolificidad natural del Ovino de Pelo Colombiano
}

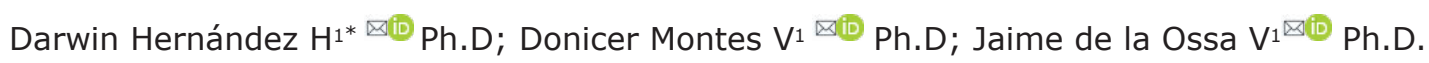

${ }^{1}$ Universidad de Sucre, Facultad de Ciencias Agropecuarias. Sincelejo. Colombia.

Correspondencia: darwin.hernandez@unisucre.edu.co

Recibido: Abril 2019; Aceptado: Julio 2019; Publicado: Diciembre 2019.

\section{RESUMEN}

Objetivo. Caracterizar y asociar el polimorfismo FecB con la prolificidad natural en los biotipos de Ovino de Pelo Colombiano (OPC) Etíope y Sudán Materiales y métodos. En 300 nacimientos provenientes de 167 ovejas de OPC, de los biotipos, Sudán $(n=73)$ y Etíope $(n=94)$, se midió el efecto del genotipo FecB, y de los factores no genéticos: número de parto de la madre, el padre, la época y el año de concepción, sobre la prolificidad natural (coderos/hembra/parto). Para esto, los animales fueron genotipados por PCR-RFLP (AvaII) para FecB y los registros productivos del rebaño fueron analizados. Se calcularon las frecuencias alélicas y genotípicas, las cuales junto con los factores no genéticos fueron asociados a la prolificidad usando un modelo GLM de efectos fijos. Resultados. El alelo FecB ${ }^{\mathrm{B}}$ presentó menor frecuencia $(0.379 \pm 0.152)$ que el alelo $\mathrm{FecB}^{+}(0.622 \pm 0.152)$ para todo el OPC. Estas frecuencias variaron ( $p<0.05$ ) entre biotipos (Sudán: 0.486 , Etíope: 0.271 ), lo mismo ocurrió con el genotipo FecBBB (0.078 en Etíope y 0.236 en Sudán). El genotipo FecB ${ }^{++}$fue más frecuente en Etíope (0.526) y el genotipo heterocigoto en Sudán $(0.5)$ y para el OPC $(0.448 \pm 0.070)$. No se encontraron diferencias significativas entre biotipos para los factores no genéticos. La prolificidad varió $(p<0.05)$ entre biotipos ( $1.45 \pm 0.22$ en Etíope y $1.34 \pm 0.03$ en Sudán), con un promedio de $1.40 \pm 0.11$ para el OPC. Ninguno de los factores no genéticos al igual que los genotipos FecB afectaron la prolificidad natural del OPC ( $p>0.05)$. Sin embargo, esta fue más alta en el genotipo FecBBB. Conclusiones. El locus estudiado fue polimórfico. La prolificidad no se afectó por los factores no genéticos ni por el genotipo FecB. Estos resultados podrían ser utilizados en planes de selección asistida para aumentar la productividad del OPC.

Palabras clave: Frecuencias alélicas, gen BMPR1, recurso genético (Fuente: $C A B$ ).

\section{ABSTRACT}

Objective. Characterize and associate the FecB polymorphism with the natural prolificacy in the biotypes of Colombian creole sheep (OPC) Etíope and Sudán. Materials and methods. At 300 births from 167 OPC sheep, from the biotypes, Sudan $(n=73)$ and Ethiopian $(n=94)$, the effect of the FecB genotype was measured, and of the non-genetic factors: number of parturitions of the mother, the father, the season and the year of conception, on the natural prolificacy (litter size). For this, the animals were genotyped by PCR-RFLP (AvaII) for FecB and the productive records of the herd analyzed. The allelic and genotypic frequencies were calculated, which, together with the

Como citar (Vancouver).

Hernández HD, Montes VD, de la Ossa VJ. Asociación del polimorfismo FecB con la prolificidad natural del Ovino de Pelo Colombiano. Rev MVZ Cordoba. 2020; 25(1):e1771. DOI: https://doi.org/10.21897/rmvz.1771 
non-genetic factors, were associated with litter size using a fixed-effect GLM model. Results. The FecB $B^{B}$ allele presented lower frequency $(0.379 \pm 0.152)$ than the FecB $B^{+}$allele $(0.622 \pm 0.152)$ for the whole OPC. These frequencies varied $(p<0.05)$ between biotypes (Sudán: 0.486, Etíope: 0.271), the same occurred with the FecBBB genotype (0.078 in Etíope and 0.236 in Sudán). The FecB ${ }^{++}$ genotype was more frequent in Etíope (0.526) and the heterozygous genotype in Sudán (0.5) and for the OPC $(0.448 \pm 0.070)$. No significant differences were found between biotypes for non-genetic factors. The prolificacy varied $(p<0.05)$ between biotypes $(1.45 \pm 0.22$ in Etíope and $1.34 \pm 0.03$ in Sudán), with an average of $1.40 \pm 0.11$ for the OPC. None of the non-genetic factors, as well as the FecB genotypes, affected the litter size of the OPC $(p>0.05)$. However, this was higher in the FecBBB genotype. Conclusions. The lucus studied was polymorphic. The litter size was not affected by non-genetic factors or the FecB genotype. These results can be used in assisted selection plans to increase OPC productivity.

Keywords: Allele frequency, BMPR1gene, genetic resources, (Source: CAB).

\section{INTRODUCCIÓN}

La principal raza ovina en Colombia es denominada Ovino de Pelo Criollo (OPC), de la cual se pueden establecer tres biotipos; Sudán, Etíope y Abisinio, con diferencias fenotípicas basadas en principalmente en el color de pelaje y tamaño $(1,2)$. En Colombia la producción ovina se realiza en sistemas tradicionales y familiares, con bajos requerimientos de insumos, los cuales están generalmente relacionados con sistemas mixtos de producción junto con otras especies como bovinos y caprinos (3). Se considera que el OPC es adecuado para la producción cárnica en sistemas extensivos, ya que la raza posee una buena adaptación a las condiciones productivas propias del clima tropical, tales como la tolerancia al calor, a ectoparásitos y la capacidad de consumir pasturas de escaso valor nutricional (4-6). Actualmente la transferencia tecnológica al sistema de producción ovino, viene desde lugares de condiciones de producción diferentes al trópico (7). Así, el mejoramiento genético en el OPC se basa en cruzamientos para aprovechar el vigor híbrido $(3,5,8)$ en características asociadas al crecimiento, pero con poco efecto sobre la eficiencia reproductiva del rebaño (9).

La eficiencia reproductiva, puede ser medida por medio de varios parámetros, entre ellos, la fertilidad, la prolificidad y la supervivencia del cordero. La prolificidad (número de corderos nacidos sobre el total de ovejas paridas) es el parámetro más estudiado. Cambios en la prolificidad se dan principalmente por un aumento en la tasa de ovulación, el número de óvulos fertilizados y la sobrevivencia embrionaria, que ocasiona un aumento del número de partos dobles y triples $(8,10)$. efectos genéticos y no genéticos. Dentro de este último factor se pueden incluir la nutrición de la cordera previa a la pubertad y al servicio, la utilización de prácticas alimenticias como el "flushing", la condición corporal de la hembra, el periodo o época del año de la monta el cual está directamente ligado a la disponibilidad de alimento, la edad cronológica de la hembra o el número de parto y diferentes tratamientos hormonales $(10,11)$. Entre los factores genéticos se pueden contar la raza, los efectos de la consanguinidad y la acción de genes únicos con efecto mayor (12). Estos son llamados genes de fecundidad (Fec) (13). Los genes Fec codifican proteínas de bajo peso molecular de función regulatoria, tanto en el desarrollo del ovario como del proceso de ovulación (14). Los tres Fec más estudiados son el Factor de Diferenciación de Crecimiento 9 (GDF9) del cual se conocen cinco polimorfismos llamados FecGI, FecGE, FecGH, FecG $^{\top}$ y FecGWNS, la Proteína Morfogenética Ósea 15 (BMP15) con ocho variantes genéticas descritas (FecX' ${ }^{\mathrm{G}}, \mathrm{Fec} \mathrm{X}^{\mathrm{H}}$, Fec $\mathrm{X}^{\mathrm{I}}$, FecXGR, FecX' FecX ${ }^{0}, F X^{B}$ y $F_{e c X^{R}}$ ) y el Receptor $1 B$ de la Proteína Morfogenética Ósea (BMPR1B) del cual solo el fenotipo denominado Boorola (FecB) ha sido descrito hasta el momento $(8,15)$.

En el OPC se han estudiado los efectos de los polimorfismos genéticos $\mathrm{FecX}^{\mathrm{R}}$, FecG ${ }^{\mathrm{H}}$ y $\mathrm{FecG}^{\mathrm{I}}$ $(8,16)$ con la prolificidad. Sin embargo, se desconocen los posibles efectos del gen BMPR1B - FecB sobre la misma. Por tanto, el objetivo de este trabajo fue caracterizar y asociar el polimorfismo FecB con la prolificidad natural en los biotipos de OPC Etíope y Sudán. 


\section{MATERIALES Y MÉTODOS}

Poblaciones, colecta de sangre y extracción

de ADN. En la presente investigación se utilizaron 167 Ovinos de Pelo Colombiano (OPC), pertenecientes a los biotipos, Sudán $(n=73)$ y Etíope $(n=94)$ de un rebaño ubicado en el departamentos de Sucre, manejados bajo condiciones de pastoreo continuo en praderas de Bothriochloa pertusa y Braquiaria brizanta, con disponibilidad de agua y sal a voluntad. Se obtuvieron muestras de sangre periférica en tubos con anticoagulante (EDTA $7.2 \mathrm{mg}$ ) teniendo en cuenta para los procedimientos de recolección de muestras, manejo y conservación, las normas éticas, técnicas, científicas y administrativas para la investigación en animales contenidas en la Ley 84 (Congreso Nacional de Colombia, 1989). El ADN se extrajo usando el estuche comercial QIAamp ${ }^{\circledR}$ DNA Mini Kit de QIAGEN. Se evaluó la cantidad y calidad del ADN usando el NanoDrop $2000^{\mathrm{TM}}$ (Thermo Fisher Scientific).

\section{Amplificación y genotipificación del locus} FecB. Se utilizó la técnica PCR-RFLP para obtener los genotipos. Primero, se amplificó un fragmento de 190pb usando los cebadores F-5 ' -CCA GAG GAC AAT AGC AAA GCA AA-3' y R-5 ' -CAA GAT GTT TTC ATG CCT CAT CAA CAC GGT C-3' (17). Las reacciones de PCR fueron llevadas a cabo en un volumen final de $25 \mu$ que contenían 20 ng de ADN, 250 nM de cada cebador y $1 X$ del súper mix MangoMix ${ }^{\mathrm{TM}}$ (Bioline(C). El perfile térmico incluyó de una desnaturalización inicial a $95^{\circ} \mathrm{C}$ durante 5 minutos, seguido por 35 ciclos de $95^{\circ} \mathrm{C}$ por $30 \mathrm{~s}, 60^{\circ} \mathrm{C}$ por $60 \mathrm{~s}$ y $72^{\circ} \mathrm{C}$ por $60 s$. Las amplificaciones fueron realizadas en un termociclador MasterCycler Nexus Gradient de Eppendorf®. La visualización de los fragmentos amplificados se realizó en electroforesis de agarosa al $1.2 \%$ teñidos con GelRed ${ }^{\mathrm{TM}}$ (Biotium). En un volumen final de $15 \mu \mathrm{l}$, que contenían en 5 $\mu l$ del producto de PCR, $1 \mathrm{U}$ de la endonucleasa de restricción AvaII (G|GACC) y 1 X de tampón, se realizaron los cortes del fragmento antes amplificado. La reacción se incubó a $37^{\circ} \mathrm{C}$ durante dos horas, seguido por $80^{\circ} \mathrm{C}$ durante 20 minutos. Los productos de digestión se visualizaron en geles al $9 \%$ de poliacrilamida (Acrilamida:Bis-acrilamida 37:1) corridos a $150 \mathrm{~V}$ por 40 minutos y teñidos con GelRed ${ }^{\mathrm{TM}}$ (Biotium). El genotipo homocigoto $\mathrm{FecB}^{++}$solo presentó un fragmento luego de la incubación (190pb), genotipo FecBBB dos fragmentos (160pb y $30 \mathrm{pb})$, mientras que, el genotipo heterocigoto $\mathrm{FecB}^{\mathrm{B}+}$ presentó tres fragmentos $(190 \mathrm{pb}, 160 \mathrm{pb}$ y $30 \mathrm{pb})(14,17,18)$.
Análisis de datos. Se calcularon las frecuencias genotípicas y alélicas para cada biotipo y en total (OPC). Estas frecuencias se compararon entre biotipos utilizando el test de Fisher con una significancia del 5\%. Todos los análisis fueron realizados con los programas Arlequin ver 3.5.2.2 (19) y GENALEX ver 6.5 (20).

A partir de los registros productivos del rebaño se calculó la prolificidad (coderos/hembra/parto) en 300 nacimientos (Etíope $n=145$ y Sudán $n=155$ ), adicionalmente, se contaron como efectos no genéticos el número de parto de la madre ( 1 al 5), la identificación del padre (1 al 6$)$, la época de concepción (seca-lluvia) y el año de concepción (2014-2018) para cada biotipo. Estos datos fueron analizados mediante estadística descriptiva y por el método de media de mínimos cuadrados se evaluaron las diferencias entre estos valores, usando el paquete estadístico $\mathrm{R} R$ (21).

Se asoció la prolificidad observada en cada biotipo y para el OPC, con los genotipos FecB encontrados utilizando el procedimiento GLM de efectos fijos, usando $R \AA$ (21) de acuerdo al siguiente modelo:

$\mathrm{Y}_{i j k l m n}=\mu+\mathrm{A}_{i}+\mathrm{B}_{j}+\mathrm{C}_{K}+\mathrm{D}_{l}+\mathrm{F}_{n}+\mathrm{H}_{m}+\varepsilon_{i j k l m n}$

Donde:

$Y_{i j k l m n}=$ prolificidad observada

$\mu=$ Efecto de la media poblacional

$\mathrm{A}_{i}=$ Efecto del i-ésimo genotipo $\mathrm{FecB}^{++}, \mathrm{FecB}^{\mathrm{B}+}$, y FecB BB

$\mathrm{B}_{j}=$ Efecto del del j-ésimo parto de la madre $j$ $=1$ al 5

$\mathrm{C}_{k}=$ Efecto del del k-ésimo padre $k=1$ al 6

$\mathrm{D}_{\text {I }}=$ Efecto del del l-ésimo año de concepción I $=2014$ al 2018

$\mathrm{F}_{n}=$ Efecto de la n-ésima epoca de concepción $n$ = seca (diciembre a abril) y lluvia (mayo a noviembre)

$\mathrm{H}_{m}=$ Efecto del m-ésimo biotipo Etíope o Sudán $\varepsilon_{i j k l n m}=$ Efecto del error aleatorio

\section{RESULTADOS}

La variante FecB en el ovino de pelo colombiano fue polimórfico, con diferencias entre biotipos (Tabla 1). El alelo $\mathrm{FecB}^{+}$presentó mayor frecuencia en el biotipo Etíope $(p<0.05)$ y el alelo $\mathrm{FecB}^{\mathrm{B}}$ en el biotipo Sudán $(\mathrm{p}<0.05)$. Para todo el OPC la frecuencia del alelo $\mathrm{FecB}^{\mathrm{B}}$ fue más baja $(0.379 \pm 0.152)$. Se encontraron diferencias significativas $(p<0.05)$ en la distribución de los 
genotipos entre los biotipos de OPC. El genotipo $\mathrm{FecB}^{++}$fue el más frecuente en el Etíope, mientras que, el genotipo $\mathrm{FecB}^{\mathrm{B}}+$ lo fue en el Sudán y en todo el OPC. El genotipo de interés $\mathrm{FecB}^{\mathrm{BB}}$ fue más frecuente en Sudán que en Etíope $(p<0.05)$, con un promedio para el OPC de $0.157 \pm 0.111$ (Tabla 1).

Tabla 1. Frecuencias alélicas y genotípicas para el locus FecB en el OPC.

\begin{tabular}{cccccc}
\hline \multirow{2}{*}{ Biotipo } & \multicolumn{2}{l}{ Frecuencias Alélicas } & \multicolumn{3}{l}{ Frecuencias Genotípicas } \\
\cline { 2 - 6 } & FecB $^{+}$ & FecB $^{\text {B }}$ & FecB $^{++}$ & FecB $^{\text {B+ }}$ & FecB $^{\text {BB }}$ \\
\hline Etíope & $0.729^{\mathrm{a}}$ & $0.271^{\mathrm{b}}$ & $0.526^{\mathrm{a}}$ & $0.396^{\mathrm{b}}$ & $0.078^{\mathrm{b}}$ \\
Sudán & $0.514^{\mathrm{b}}$ & $0.486^{\mathrm{a}}$ & $0.264^{\mathrm{b}}$ & $0.500^{\mathrm{a}}$ & $0.236^{\mathrm{a}}$ \\
& 0.622 & 0.379 & 0.395 & 0.448 & 0.157 \\
OPC & \pm 0.152 & \pm 0.152 & \pm 0.185 & \pm 0.070 & \pm 0.111 \\
\hline
\end{tabular}

a,bLetras diferentes en la misma columna, difieren estadísticamente $(p<0.05)$.

En las variables número de parto de la madre, padre, época de concepción y año de concepción, no se encontraron diferencias estadísticas entre los biotipos. Por tanto, se presenta la estadística descriptiva de estos valores como promedios para el OPC (Tabla 2). En promedio cada hembra tuvo $2.7 \pm 1.5$ partos. La mayoría de las hembras tuvo un solo parto (39.5\%), el $20.7 \%, 12 \%, 10 \%$ y $9 \%$ tuvieron dos, tres, cuatro y cinco partos respectivamente. Las hembras fueron servidas por seis machos, los cuales sirvieron en promedio $15 \pm 6.4$ hembras cada uno. En promedio, en cada época se sirvieron $70 \pm 5.6$ hembras en cinco años con promedio de $35 \pm 11$ montas/año.

Tabla 2. Estadística descriptiva para las variables evaluadas en la raza OPC.

\begin{tabular}{|c|c|c|c|c|}
\hline Variable & Promedio \pm D.E. & Mínimo & Máximo & CV \\
\hline Prolificidad $^{a}$ & $1.40 \pm 0.11$ & 1 & 2 & $7.9 \%$ \\
\hline Parto de la madre ${ }^{b}$ & $2.7 \pm 1.5$ & 1 & 5 & $55.6 \%$ \\
\hline Padre $^{c}$ & $15 \pm 6.4$ & 10 & 25 & $42.7 \%$ \\
\hline Época de concepción ${ }^{d}$ & $70 \pm 5.6$ & 65 & 76 & $8.0 \%$ \\
\hline Año de concepción ${ }^{e}$ & $35 \pm 11$ & 27 & 48 & $31.4 \%$ \\
\hline
\end{tabular}

El número de parto de la madre, el efecto del padre, de la época de concepción y año de concepción no afectaron ( $p>0.05$ ) la prolificidad en los biotipos de OPC estudiados. Por otro lado, la prolificidad promedio sí varió significativamente entre biotipos $(p<0.05)$ con valor de $1.45 \pm 0.22$ en Etíope y $1.34 \pm 0.03$ para Sudán (Tabla 3).
Tabla 3. Prolificidad promedio entre genotipos FecB para cada biotipo.

\begin{tabular}{ccccc}
\hline \multirow{2}{*}{ Biotipo } & \multicolumn{3}{c}{ Genotipos } & Promedios \\
\cline { 2 - 5 } & FecB $^{++}$ & FecB $^{\text {B+ }}$ & FecBBB $^{\text {B }}$ & \\
\hline Etíope & $1.2 \pm 0.4$ & $1.56 \pm 0.6$ & $1.60 \pm 0.5$ & $1.45 \pm 0.22^{\mathrm{a}}$ \\
Sudán & $1.33 \pm 0.5$ & $1.32 \pm 0.4$ & $1.38 \pm 0.4$ & $1.34 \pm 0.03^{\mathrm{b}}$ \\
OPC & $1.27 \pm 0.09$ & $1.44 \pm 0.1$ & $1.49 \pm 0.1$ & $1.40 \pm 0.11$ \\
\hline
\end{tabular}

a,bLetras diferentes en la misma columna, difieren estadísticamente $(p<0.05)$.

Para toda la población la prolificidad promedio encontrada fue de $1.40 \pm 0.11$ con un valor máximo de 2 crías/hembra/parto.

La asociación ente genotipo y prolificidad no fue significativa (Tabla 3), aunque, esta fue mayor en el genotipo $\mathrm{FecB}^{\mathrm{BB}}$ en los dos biotipos y para todo el OPC, respecto de los otros genotipos.

\section{DISCUSIÓN}

La variante $\mathrm{FecB}$ consiste en el cambio de una Adenina por una Guanina (c830A>G), lo que ocasiona el cambio no sinónimo en posición 249 de la proteína, cambiando la Glutamina por Arginina (Q249R) $(14,15,22,23)$. Este es el primer reporte de la presencia de la mutación $\mathrm{FecB}^{\mathrm{B}}$ en el OPC. En otras razas en el mundo la frecuencia de este alelo fue de 0.35 en Kalehkoohi (22), 0.14 en Nilagiri (17), 0.61 en Garole (24), 1.0 en Hu (18), 0.63 en Han (18) y 0.5 en Bayanbulak (25). Mientras que, este alelo no se ha encontrado en las razas Barbarine, Queue Fine de L'Ouest, Noire de Thibar, SiciloSarde, D'man, Romanov, Finn, East Friesian, Teeswater, Blueface Leicester, D'Man, Chios, Mountain Sheep, German Whiteheaded Mutton, Lleyn, Loa, Galician, Barbados Blackbelly y St. Croix $(18,16)$.

El tamaño de la camada tiene un gran impacto en la eficiencia reproductiva de las ovejas. La prolificidad, está directamente relacionada con la tasa de ovulación (22) que es el resultado de la variación en la sensibilidad de la liberación de gonadotropinas y a los efectos de retroalimentación de los esteroides gonadales (27). Estas pueden ser afectadas tanto por factores genéticos ( $\mathrm{FecB}$ ) como no genéticos (8). La prolificidad promedio encontrada en esta investigación $(1.40 \pm 0.11)$ fue más alta que lo reportado por otros autores para este mismo grupo racial $(4,8,28)$. 
Diversos autores muestran un efecto significativo $(p<0.01)$ en el aumento de la prolificidad en las ovejas con genotipo $\mathrm{FecB}^{\mathrm{BB}}(15,18,22,23)$. Así, el gen BMPR1B es uno de los genes candidatos clave para el control genético de la tasa de ovulación y el consiguiente aumento de la prolificidad en diferentes razas ovinas. Se ha demostrado que el alelo mutado (FecB ${ }^{\mathrm{B}}$ ) produce un aumento en la tasa de ovulación, que, adicionalmente aumenta entre +1 a +1.5 óvulos liberados por cada copia del alelo FecB $^{B}$ (29). Luego, en promedio, las ovejas con genotipo $\mathrm{FecB}^{\mathrm{BB}}$ o heterocigotas $\left(F \mathrm{CB}^{\mathrm{B}+}\right.$ ) tienen entre 0.64 y 0.35 más crías al parto, respectivamente, que las ovejas con genotipo $\mathrm{FecB}^{++}$. Lo anterior convierte al alelo $F_{e c B}{ }^{B}$ y al genotipo $F_{e c B}{ }^{B B}$ como objetos de interés zootécnico.

En este trabajo no se encontró una asociación significativa entre genotipo y prolificidad, sin embargo, en ambos biotipos y en general para el OPC el genotipo FecB $^{B B}$ presentó la mayor prolificidad. Lo cual representó 0.4 y 0.05 más crías en Etíope y Sudán, respectivamente. Para el OPC este aumento en la prolificidad fue de $14.7 \%$.

El biotipo Sudán presentó mayor frecuencia del alelo y del genotipo de interes $\left(\mathrm{FecB}^{\mathrm{B}}\right.$ y FecB $\left.{ }^{B B}\right)$ respecto al biotipo Etíope $(p<0.05)$, sin embargo, la prolificidad observada fue mayor en el biotipo Etíope $(p>0.05)$. Lo anterior sugiere, que la prolificidad natural para la raza OPC, está seguramente bajo el control de otros genes no determinados hasta el momento. Al respecto, se han genotipado y asociado los polimorfismos genéticos FecXR, FecGH y FecG $^{I}(8,16)$ con la prolificidad en el OPC, con resultados no significativos.

Al igual que en este trabajo, Pineda et al (8) reportan que los factores clasificados como no genéticos (número de parto de la madre, padre, de la época y año de concepción) no afectaron $(p>0.05)$ la prolificidad en la población de OPC estudiada.

Varios autores reportan que la prolificidad natural de la oveja aumenta con la edad del animal $(4,30,31)$. Una posible explicación al fenómeno puede atribuirse a la edad (32) y al peso corporal de la oveja al momento de apareamiento, ya que conforme la oveja madura y alcanza su desarrollo corporal y fisiológico, se hace más eficiente para mantener una gestación, producir más leche y expresar su habilidad materna (33). El crecimiento compite con la gestación y los procesos reproductivos por la obtención de nutrientes circulantes en el organismo, lo que disminuye los parámetros reproductivos. Así es, como sucede en la mayoría de los sistemas de producción extensivos tropicales, donde el sistema de apareamiento es continuo y las ovejas son apareadas generalmente entre los 20 y 26 $\mathrm{kg}(34)$.

El efecto del padre no afecto la prolificidad de la hembra OPC, estas diferencias en el efecto del macho, pueden estar íntimamente relacionadas con la condición corporal, el estado nutricional, estado sanitario, la proporción machos:hembra en los lotes de monta y descanso del macho entre épocas o estaciones de monta, las cuales repercuten de una u otra manera sobre la calidad espermática y la función reproductiva $(8,35)$.

Las dos variables relacionadas con la fecha de concepción de las crías no tuvieron relación significativa con la prolificidad, aunque el año 2014 y en la época de lluvia esta fue mayor. En ovinos Pelibuey $x$ Blackbelly y sus cruces con Dorper y Katahdin en México reportaron efecto significativo del año, pero no de la época sobre la prolificidad (31). En la raza Lacaune encontró efecto del año (35).

\section{Conflicto de intereses.}

Los autores del presente estudio declaramos que no existe conflicto de intereses con la publicación de este manuscrito.

\section{Agradecimientos}

A la Universidad de Sucre por la financiación de esta investigación y al productor de OPC en el departamento de Sucre que permitió la colecta de las muestras. 


\section{REFERENCIAS}

1. Flórez JM, Hernández M de J, Bustamante M, Vergara OD. Caracterización morfoestructural de tres poblaciones de Ovino de Pelo Criollo Colombiano "OPC." Archivos de Zootecnia. 2018; 67(259):340-8. DOI: https://doi. org/10.21071/az.v67i259.3789

2. Montes D, Moreno J, Lugo N, Ramirez R, Celis A, Garay G. Caracterización Faneróptica y Morfológica de la hembra ovina de pelo criollo (Camura) colombiana, en la sub región Sabanas y Golfo de Morrosquillo Departamento de Sucre. Revista Colombiana de Ciencias Pecuarias. 2013; 5(1):104-15. DOI: https://doi.org/10.24188/recia. v5.n1.2013.475

3. Ocampo R, Martínez R, Rocha J, Cardona $\mathrm{H}$. Genetic characterization of Colombian indigenous sheep. Revista Colombiana de Ciencias Pecuarias. 2017; 30(2):116-25. DOI: http://dx.doi.org/10.17533/udea. rccp.v30n2a03

4. Cuellar-Gamboa G, Jimenez-Robayo L, Grajales-Lombana H, Morales-Mendoza L, Leal-Gutierrez J, Sanchez-Isaza C. Factores que influencian la prolificidad en ovinos del centro agropecuario marengo, Colombia. Actas Iberoamericanas de Conservación Animal. 2015; 6(1):460-5. URL Available in: https://aicarevista. jimdo.com/app/download/11698017525/ AICA2014 Trabajo011.pdf?t $=1542558897$

5. Montes D, Lenis C, Hernández D. Polymorphisms of the calpain and calpastatin genes in two populations of colombian creole sheep. Revista MVZ Córdoba. 2019; 24(1):7113-8. DOI: https://doi. org/10.21897/rmvz.1345

6. Carrillo D, Hernández D. Characterization seminal of individuals hair sheep colombian creole in the department of Sucre. Revista Colombiana Ciencia Animal. 2016; 8(2):197203. DOI: https://doi.org/10.24188/recia. v8.n2.2016.187

7. Ospina O, Grajales H, Manrique C. Gestión del conocimiento: mayor producción y competitividad. Perspectivas para los sistemas de producción ovino-caprinos. Rev Med Vet 2011; 22:95-113. https://doi. org/10.19052/mv.564
8. Pineda R, Montes D, Hernández D. Association of the polymorphisms FecX ${ }^{\mathrm{R}}$, FecGH, and FecG ${ }^{I}$ and non-genetic factors that affect the prolificacy of colombian creole sheep. Indian Journal of Science and Technology. 2018 ;11(17). DOI: http://dx.doi.org/10.17485/ ijst/2018/v11i17/122374

9. Simanca J, Vergara O, Bustamante $M$. Description of growth in sheep creole (Ovis aries) in two populations from Córdoba, Colombia. Rev MVZ Córdoba. 2017; 22(3):6310-9. DOI: https://doi. org/10.21897/rmvz.1135

10. Alabart JL, Lahoz B, Calvo JH, Jurado JJ, Fantova $\mathrm{E}$, Equipo Técnico de UPRA-Grupo Pastores, J Folch. Estudios realizados y situación actual de la variante génica prolífica ROA (FecXR) de la raza ovina Rasa Aragonesa. Archivos de Zootecnia. 2016; 65(251):449-52. DOI: https://doi. org/10.21071/az.v65i251.714

11. Vicente-Pérez R, Avendaño-Reyes L, Álvarez FD, Correa-Calderón A, Meza-Herrera $C A$, Mellado $M$, et al. Comportamiento productivo, consumo de nutrientes y productividad al parto de ovejas de pelo suplementadas con energía en el preparto durante verano e invierno. Archivos de medicina veterinaria. 2015; 47(3):301-9. DOI: http://dx.doi.org/10.4067/S0301$\underline{732 \times 2015000300006}$

12. Vera $M$, Aguion M, Bouza C. Detection of Grivette BMP15 prolificacy variant (FecXGR) in different sheep breeds presented in Galicia (NW Spain). Gene Reports. 2018; 12:109-14. DOI: https://doi.org/10.1016/j. genrep.2018.06.008

13. Luna C, Alonso R. Genes con efecto mayor sobre la fertilidad de ovejas. Revisión. Revista Mexicana de Ciencias Pecuarias. 2014; 5(1):107-30. URL Available in: https://cienciaspecuarias.inifap.gob. $\mathrm{mx} /$ index.php/Pecuarias/article/view/3219

14. Ahlawat $S$, Sharma R, Roy M, Mandakmale S, Prakash V, Tantia M. Genotyping of novel SNPs in BMPR1B, BMP15, and GDF9 genes for association with prolificacy in seven indian goat breeds. Animal Biotechnology. 2016; 27(3):199-207. DOI: https://doi.or $\mathrm{g} / 10.1080 / 10495398.2016 .1167706$ 
15. Albarella A, Ciotola F, Selvaggi M, Dario C, Licciardi S, Scopino G, et al. Analysis of major fecundity genes in autochthonous Laticauda and Bagnolese sheep breeds. Small Ruminant Research. 2015; 133:11822. DOI: https://doi.org/10.1016/j. smallrumres.2015.09.005

16. Hernández $D$, Montes $D$, Otero $R$, Prieto E, Espitia A, Buelvas M. Polimorfismos FecXR, FecGI y FecGH en el ovino de pelo colombiano. Archivos de Zootecnia. 2019; 68(262):192-7. DOI: https://doi. org/10.21071/az.v68i262.4136

17. Sudhakar A, Rajendran R, Rahumathulla PS. Detection of Booroola (FecB) mutation in Indian sheep-Nilagiri. Small Ruminant Research. 2013; 113(1):557. DOI: https://doi.org/10.1016/j. smallrumres.2013.02.012

18. Davis $\mathrm{GH}$, Balakrishnan L, Ross IK, Wilson T, Galloway SM, Lumsden BM, et al. Investigation of the Booroola (FecB) and Inverdale (FecXI) mutations in 21 prolific breeds and strains of sheep sampled in 13 countries. Animal Reproduction Science. 2006; 92(1):87-96. DOI: http://10.1016/ j.anireprosci.2005.06.001

19. Excoffier L, Lischer HEL. Arlequin suite ver 3.5: a new series of programs to perform population genetics analyses under Linux and Windows. Mol Ecol Resour. 2010; 10(3):564-7. DOI: https://doi.org/10.1111/ j.1755-0998.2010.02847.x

20. Peakall R, Smouse PE. GenAlEx 6.5: genetic analysis in Excel. Population genetic software for teaching and research--an update. Bioinformatics. 2012; 28(19):25372539. DOI:https://doi.org/10.1093/ bioinformatics/bts460

21. R Core Team. R: A language and environment for statistical computing [Internet]. Vienna, Austria: R Foundation for Statistical Computing; 2014. URL Available in: http:// www.R-project.org/

22. Mahdavi M, Nanekarani S, Hosseini S. Mutation in BMPR-IB gene is associated with litter size in Iranian Kalehkoohi sheep. Animal Reproduction Science. 2014; 147(3):93-98. DOI: https://doi. org/10.1016/j.anireprosci.2014.04.003
23. Wilson $T$, Wu X, Juengel J, Ross I, Lumsden J, Lord $E$, et al. Highly prolific Booroola sheep have a mutation in the Intracellular Kinase Domain of Bone Morphogenetic Protein IB Receptor (ALK-6) that is expressed in both oocytes and granulosa cells. Biology Reproduction. 2001; 64(4):12251235. DOI: https://doi.org/10.1095/ biolreprod64.4.1225

24. Polley $S$, De $S$, Brahma B, Mukherjee $A$, Vinesh P, Batabyal S, et al. Polymorphism of BMPR1B, BMP15 and GDF9 fecundity genes in prolific Garole sheep. Tropical Animal Health and Production. 2010; 42(5):985-93. DOI: http://10.1007/s11250-009-9518-1

25. Zuo B, Quian H, Wang Z, Wang X, Nisa N, et al. A Study on BMPR-IB Genes of Bayanbulak Sheep. Asian-Australasian J Animal Sci. 2013; 26(1):36-42. DOI: https://doi. org/10.5713/ajas.2012.12238

26. Vacca G, Dhaouadi A, Rekik M, Carcangiu V, Pazzola M, Dettori M. Prolificacy genotypes at BMPR 1B, BMP15 and GDF9 genes in North African sheep breeds. Small Ruminant Research. 2010; 88(1):6771. DOI: https://doi.org/10.1016/j. smallrumres.2009.11.005

27. Miao X, Luo Q, Zhao H, Qin X. Ovarian transcriptomic analysis reveals the alternative splicing events associated with fecundity in different sheep breeds. Animal Reproduction Science. 2018; 198:177183. DOI: https://doi.org/10.1016/j. anireprosci.2018.09.017

28. Rodriguez M, López A, Grajales H, Naranjo A. Evaluación de parámetros productivos y reproductivos en cruces de cuatro razas ovinas. Revista Medicina Veterinario y Zootecnia. 1995;43(1):14-18. URL Available in: https://revistas.unal.edu.co/index.php/ remevez/article/view/47559/48654

29. Fabre S, Pierre A, Mulsant P, Bodin L, Di Pasquale $E$, Persani $L$, et al. Regulation of ovulation rate in mammals: contribution of sheep genetic models. Reprod Biol Endocrinol. 2006; 4(20):1-12. DOI: https:// doi.org/10.1186/1477-7827-4-20

30. Liandris $E$, Kominakis $A$, Andreadouna $M$, Kapeoldassia K, Chadio S, Tsiligiannic T, Gazouli M, Ikonomopoulosa I. Associations between single nucleotide polymorphisms of GDF9 and BMP15 genes and litter size in two dairy sheep breeds of Greece. Small Rumin Res. 2012; 107(1):16-21. DOI: https://doi. org/10.1016/j.smallrumres.2012.04.004 
31. Hinojosa-Cuéllar J, Oliva-Hernández J, Torres-Hernández G, Segura-Correa J. Comportamiento productivo de corderos F1 Pelibuey x Blackbelly y cruces con Dorper y Katahdin en un sistema de producción del trópico húmedo de Tabasco, México. Archivos de Medicina Veterinaria. 2013; 45(2):135-143. DOI: http://dx.doi. org/10.4067/S0301-732X2013000200004

32. Mohammadabadi M, Sattayimokhtari R. Estimation of (co) variance components of ewe productivity traits in Kerman sheep. Slovakia Journal of Animal Science. 2013; 46(2):45-51. URL Available in: http://www. vuzv.sk/slju/13 2/2Mohammadabadi.pdf

33. Hinojosa-Cuellar J, Torres-Hernández G, Oliva-Hernández J, Aranda-Ibañez E, Segura-Correa J, González-Camacho J. Pre-weaning performance of lambs from purebred and crossebred hair ewes under hunid tropical conditions of tabasco, Mexico. J Anim Vet Adv. 2011; 10(23):3149-3154. DOI: http://10.3923/javaa.2011.3149.3154
34. Magaña-Monforte J, Huchin-Cab M, AkeLópez R, Segura-Correa J. A field study of reproductive performance and productivity of Pelibuey ewes in Southeastern Mexico. Trop Anim Health Prod. 2013; 45(8):17711776. DOI: http://10.1007/s11250-013$\underline{0431-2}$

35. SanCristobal-Gaudy M, Bodin L, Elsen J, Chevalet C. Genetic components of litter size variability in sheep. Genet Sel Evol. 2001; 33(3):249-271. DOI: https://doi. org/10.1186/1297-9686-33-3-249 